Coher ent backscattering in a di sorder ed opt i cal medi umin the presence of sat urat $i$ on absor pt i on

\begin{tabular}{|l|l|}
\hline 著者 & Tot suka Kouki, Tomi t a Nakot o \\
\hline $\begin{array}{l}\text { j our nal or } \\
\text { publ i cat i on t i t l e }\end{array}$ & Physi cal Revi ew B \\
\hline vol une & 59 \\
\hline number & 17 \\
\hline page range & $11139-11142$ \\
\hline year & 1999- 05 \\
\hline 出版者 & Aner i can Physi cal Soci et y \\
\hline 権利 & (c) 1999 The Aner i can Physi cal Soci et y \\
\hline URL & ht t p: //hdl . handl e. net /10297/578 \\
\hline
\end{tabular}




\title{
Coherent backscattering in a disordered optical medium in the presence of saturation absorption
}

\author{
Kouki Totsuka and Makoto Tomita \\ Department of Physics, Faculty of Science, Shizuoka University, 836 Ohya, Shizuoka City 422, Japan
}

(Received 23 October 1998)

\begin{abstract}
We theoretically describe the coherent backscattering peak in a disordered medium in the presence of saturation absorption. When the excitation intensity is increased above the saturation intensity, the reflectivity of the incident light increases and the width of the peak becomes narrow. This effect is evident when the absorption length is comparable to the transport mean free path. The effect of the saturation absorption on the peak is compared with the effects of the normal absorption and the confined geometry.

[S0163-1829(99)02018-4]
\end{abstract}

Weak localization is a precursor of Anderson localization, which means waves are localized in disordered media when the disorder becomes a certain critical strength. The microscopic mechanism of the weak localization is the constructive interference of waves in time-reversed paths. In microelectronics, this effect explains the negative magnetic resistance and the dependence of the resistance on the sample temperature. In optics, the effect of the weak localization directly appears as a coherent backscattering peak. The scattered intensity is two times as high as the background intensity in the backward direction within a range of $\lambda / l^{*}$, where $\lambda$ is the wavelength, and $l^{*}$ is the transport mean free path. Various experimental and theoretical studies have been reported for the coherent backscattering peak. ${ }^{1-8}$ Etemad examined the effect of the absorption and the confined geometry and classified the trajectories. ${ }^{9}$ Absorption and the confined geometry remove long trajectories in the medium and makes the top of the coherent backscattering peak rounded off. Wiersma has reported that the shape of the coherent backscattering is sharpened in an amplifying disordered medium since long paths are more amplified in the medium. ${ }^{10}$ In such a scattering and amplifying medium interference effect among long paths becomes more important and localization of the photon is expected to occur.

In this paper, we theoretically describe the coherent backscattering peak in the disordered medium in the presence of the saturation absorption. Saturation absorption could be an important phenomenon in the disordered medium. Genack discussed that the path length calculated on the basis of a simple diffusion picture is not enough to explain the gainpath length product for the random laser and suggested that the saturation absorption could be the mechanism to explain the large gain-path length product in the disordered medium. ${ }^{11}$ It is also reported that the reflectivity from absorptive medium shows a nonlinear behavior. ${ }^{12}$ The reflectivity increases when the excitation intensity is increased above the saturation intensity since the incident light is scattered out from the medium before it is absorbed. The excitation power-dependent photoluminescence spectra in dyes in strongly scattering media were also explained by the saturation absorption in the excitation process and the reabsorption in the luminescence process. ${ }^{13}$ The effect of the saturation absorption could appear in a variety of phenomena in the disorder medium, such as statistics in the fluctuations, transmittance, time spreading, and the dynamics in scattering. Here, we discuss the intensity and shape of the coherent backscattering peak in the disordered medium in the presence of the saturation absorption.

We consider the reflected light from a semi-infinite absorptive disordered medium which occupies the region $z$ $>0$. The incident plane wave with a wave vector $\mathbf{k}_{i}$ comes from the half space $z<0$ and illuminates the disordered medium. The albedo per unit solid angle around the direction $\mathbf{k}_{i}$ is expressed as ${ }^{3}$

$$
\begin{aligned}
\alpha\left(\mathbf{k}_{i}, \mathbf{k}_{f}\right)= & \frac{c}{4 \pi l^{* 2}} \int_{0}^{\infty} \int_{0}^{\infty} e^{-z_{+} / \mu l_{t}} e^{-z_{-} / \mu^{\prime} l_{t}} \\
& \times \int_{\rho}\left(1+e^{-i \mathbf{q} \cdot\left(\mathbf{r}-\mathbf{r}^{\prime}\right)}\right) Q\left(\mathbf{r}, \mathbf{r}^{\prime}\right) d^{2} \rho d z_{+} d z_{-} .
\end{aligned}
$$

Here, $c$ is the velocity of light, $z_{+}$and $z_{-}$are projections of $\mathbf{r}$ and $\mathbf{r}^{\prime}$ on the $z$ axis, $\mu$ and $\mu^{\prime}$ are the projections of $\mathbf{k}_{i}$ and $\mathbf{k}_{f}$ on the $z$ axis, $l_{t}$ is the transport mean path which is related to the elastic transport mean free path $l^{*}$ and the absorption length $l_{a}$ as $l_{t}^{-1}=l^{*-1}+l_{a}^{-1}, \mathbf{q} \equiv \mathbf{k}_{i}+\mathbf{k}_{f}, \boldsymbol{\rho}$ is the projection of $\mathbf{r}-\mathbf{r}^{\prime}$ on the incident surface, and $Q\left(\mathbf{r}, \mathbf{r}^{\prime}\right)$ is the propagator which describes the light transport from an incident point $\mathbf{r}$ to an outgoing point $\mathbf{r}^{\prime}$. Assuming the normal incidence and the backscattering light at a small angle from the direction normal to the incident surface, and the incident point $\mathbf{r}$ and the outgoing point $\mathbf{r}^{\prime}$ is on the same plane $z$ $=l_{t}$, the albedo Eq. (1) as a function of $\theta$ becomes

$$
\alpha(\theta)=\frac{c l_{t}^{2}}{4 \pi l^{* 2}} \int_{\rho} d^{2} \rho\left(1+e^{-i \rho \cdot q_{\perp}}\right) Q\left(\mathbf{r}, \mathbf{r}^{\prime}\right) .
$$

Here, $\mu \fallingdotseq \mu^{\prime} \fallingdotseq 1, \mathbf{q}_{\perp}$ is the projection of $\mathbf{q}$ on the incident surface with $\left|\mathbf{q}_{\perp}\right| \fallingdotseq 2 \pi \theta / \lambda$. Therefore, the interference term of the coherent backscattering peak is given by the Fourier transform of $|\boldsymbol{\rho}| Q\left(\mathbf{r}, \mathbf{r}^{\prime}\right)$ on the plane $z=l_{t}$ and the angular width is about $\lambda / 2 \pi l^{*}$.

We analyze the light propagation in the medium with the saturation absorption. Saturation absorption is a universal phenomenon. Basically, it is observed in two-level systems. Here we consider a three-level system as a realistic system where the saturation absorption is observed. Other systems, 
such as the simple two-level system or the complicated fourlevel system could be treated in a similar manner. Let us consider dye molecules, for which we discuss numerical estimation for the experiments, as an example of a three-level system. The incident light excites the absorbers from the ground state $S_{0}$ into the excited state $S_{2}$. The population in the state $S_{2}$ decays into the $S_{1}$ state just below the $S_{2}$ state through the fast nonradiative decay process. The population in the $S_{1}$ state decays to the ground state, $S_{0}$ with a lifetime of $\tau$. The rate equation for this system is

$$
\frac{\partial N_{1}(\mathbf{r}, t)}{\partial t}=\sigma N_{0}(\mathbf{r}, t) I(\mathbf{r}, t)-\frac{N_{1}(\mathbf{r}, t)}{\tau},
$$

where $N_{0}(\mathbf{r}, t)$ and $N_{1}(\mathbf{r}, t)$ are populations in the ground state $S_{0}$ and the excited state $S_{1}$, respectively, and are related to the total population of the absorber $N$ as $N=N_{0}(\mathbf{r}, t)$ $+N_{1}(\mathbf{r}, t) . \sigma$ is the absorption cross section for incident light. $I(\mathbf{r}, t)$ is the intensity of the incident light. The propagation of the light in the medium is analyzed on the basis of the diffusion equation

$$
\frac{\partial I(\mathbf{r}, t)}{\partial t}=\frac{l^{*} c}{3} \nabla^{2} I(\mathbf{r}, t)-c \sigma N_{0}(\mathbf{r}, t) I(\mathbf{r}, t) .
$$

Let us consider the steady-state condition: $\partial N_{1}(\mathbf{r}, t) / \partial t$ $=0, \partial I(\mathbf{r}, t) / \partial t=0$. When the intensity of the incident light is weak, the ground-state population is approximated as $N_{0}$ $=N$. The steady-state solution of Eq. (4) is

$$
Q_{0}\left(\mathbf{r}-\mathbf{r}^{\prime}\right)=\frac{1}{4 \pi D\left|\mathbf{r}-\mathbf{r}^{\prime}\right|} \exp \left(-\frac{\left|\mathbf{r}-\mathbf{r}^{\prime}\right|}{\xi}\right) .
$$

Here, $D=c l^{*} / 3$ is the diffusion coefficient, $\xi=\sqrt{l^{*} l_{a} / 3}$ is the mean penetration depth of light. For the semi-infinite sample, the propagator $Q\left(\mathbf{r}, \mathbf{r}^{\prime}\right)$ is expressed as

$$
Q\left(\mathbf{r}-\mathbf{r}^{\prime}\right)=Q_{0}\left(\mathbf{r}-\mathbf{r}^{\prime}\right)-Q_{0}\left(\mathbf{r}-\mathbf{r}^{\prime *}\right),
$$

where $\mathbf{r}^{\prime *}$ is the mirror image of $\mathbf{r}^{\prime}$ in the trapping plane $z_{0}$ located on $z_{0}=-0.7 l^{*}$.

When the intensity of the incident light is increased, the saturation in the absorption process becomes important. The steady-state solution of the rate equation is

$$
N_{0}(\mathbf{r})=N\left(\frac{1}{1+I(\mathbf{r}) / I_{s}}\right),
$$

where $I_{s}=1 /(\sigma \tau)$ is the saturation intensity. We calculate $Q\left(\mathbf{r}, \mathbf{r}^{\prime}\right)$ using the numerical method. The procedure is as follows. The excitation light intensity $I(\mathbf{r})$ and the local absorption coefficient are calculated for the infinite beam size plane wave. Figure 1 shows the normalized spatial distribution of the excitation light $I(\mathbf{r})$ and the local absorption coefficient, respectively, as a function of $z$ for four different incident light intensities. When an intensity is increased, the incident light penetrates into the deeper region and the local absorption coefficient decreases in the medium. In the presence of saturation absorption, $l_{t}$ also depends on the incident light intensity. Then, a source point is set at a position $\mathbf{r}^{\prime}$ $=\left(0,0, l_{t}\right)$. The propagator $Q\left(\mathbf{r}, \mathbf{r}^{\prime}\right)$ is calculated as the solution of the diffusion equation with the $z$-dependent absorption coefficient shown in Fig. 1. The shape of the coherent

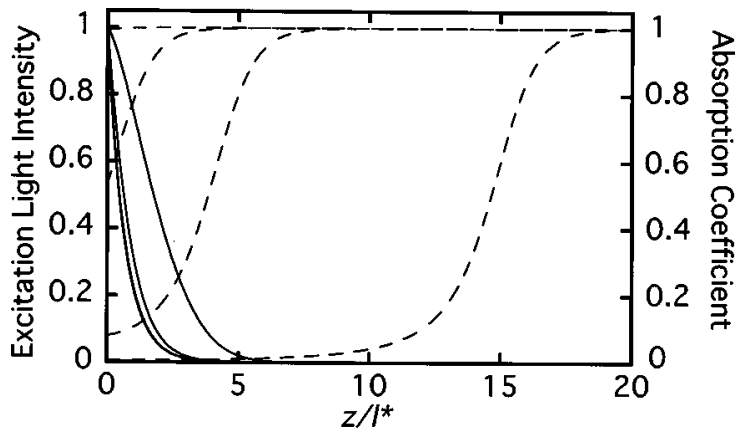

FIG. 1. Solid and dashed lines are calculated curves for the excitation light intensity and the local absorption coefficient for $l_{a}$ $=l^{*}$ for four different excitation intensities, $0.7 I_{S}, 0.7 \times 10^{2} I_{S}$, $0.7 \times 10^{3} I_{S}$, and $0.7 \times 10^{4} I_{S}$. The horizontal axis is the distance from the incident surface normalized by the transport mean path $l^{*}$. The solid lines indicate the increase of the penetration depth with increasing excitation intensity and the dashed lines indicate the decrease of the absorption coefficient with increasing excitation intensity. The spatial distribution of the excitation light intensity at 0.7 $\times 10^{2} I_{S}$ is almost the same at $0.7 I_{S}$.

backscattering peak is calculated as the Fourier transform of $|\boldsymbol{\rho}| Q\left(\mathbf{r}, \mathbf{r}^{\prime}\right)$. Figure 2 shows $\left|\mathbf{r}-\mathbf{r}^{\prime}\right| Q\left(\mathbf{r}, \mathbf{r}^{\prime}\right)$ for the sample $l_{a}=l^{*}$ for two different excitation intensities. Figures 2(a) and 2 (b) are for $0.7 I_{s}$ and $0.7 \times 10^{4} I_{s}$, respectively. When the intensity is weak $\left|\mathbf{r}-\mathbf{r}^{\prime}\right| Q\left(\mathbf{r}, \mathbf{r}^{\prime}\right)$ shows exponential behavior which is characteristic to the absorption process. With increasing intensity, $\left|\mathbf{r}-\mathbf{r}^{\prime}\right| Q\left(\mathbf{r}, \mathbf{r}^{\prime}\right)$ shows linear behavior in the region $z / l^{*}<5$, reflecting that the diffusion is the main
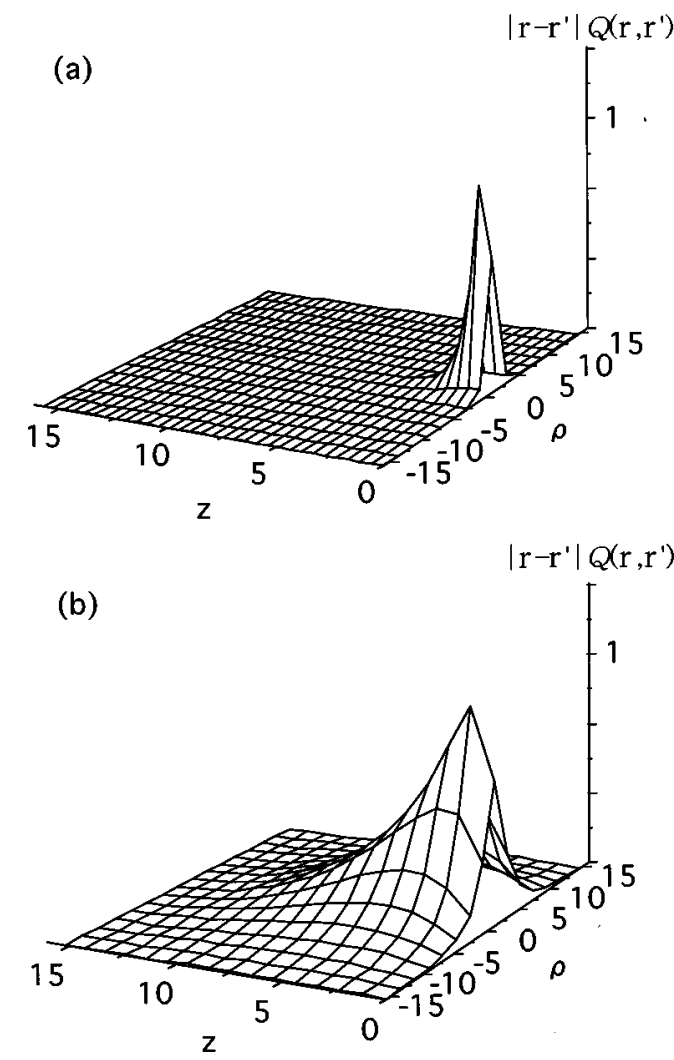

FIG. 2. Normalized spatial distribution of $\left|\mathbf{r}-\mathbf{r}^{\prime}\right| Q\left(\mathbf{r}, \mathbf{r}^{\prime}\right)$ for $l_{a}=l^{*}$ for two different excitation intensities: (a) $0.7 I_{S}$ and (b) $0.7 \times 10^{4} I_{S}$. 

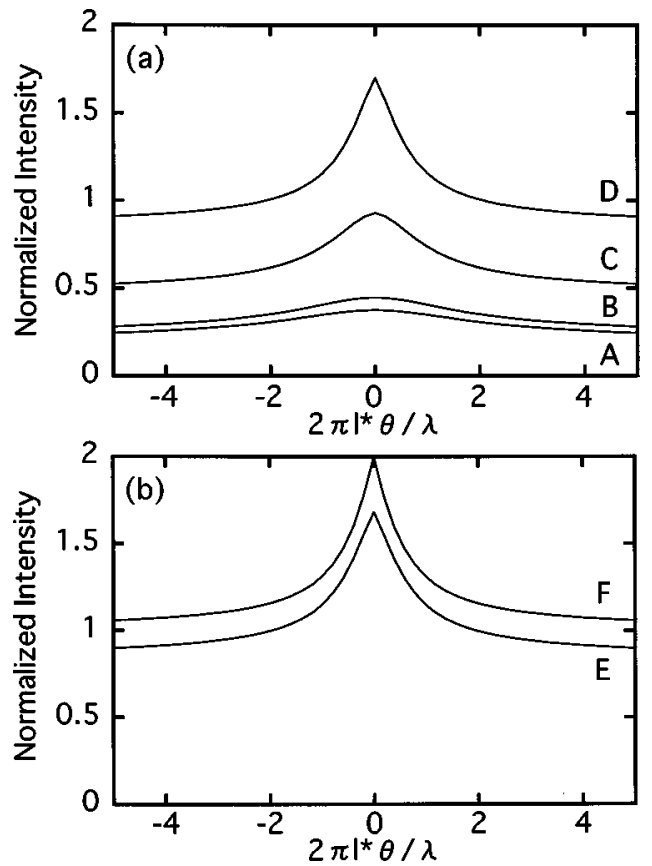

FIG. 3. Coherent backscattering peaks at different excitation intensities for two different absorption lengths: (a) The absorption length is $l_{a}=l^{*}$ and the excitation intensities for these curves are (A) $0.7 I_{S}$; (B) $0.7 \times 10^{2} I_{S}$; (C) $0.7 \times 10^{3} I_{S}$; and (D) $0.7 \times 10^{4} I_{S}$. (b) The absorption length is $l_{a}=100 l^{*}$ and the excitation intensities for these curves are (E) $0.7 I_{S}$ and $(\mathrm{F}) 0.7 \times 10^{4} I_{S}$.

mechanism to determine the shape of $\left|\mathbf{r}-\mathbf{r}^{\prime}\right| Q\left(\mathbf{r}, \mathbf{r}^{\prime}\right)$ at an intensity above the $I_{s}$. Figure 3(a) shows the calculated curves of the coherent backscattering peaks for the sample $l_{a}=l^{*}$ for four different intensities. At weak intensity the shape of the coherent backscattering peak is rounded off, which is a characteristic for the absorptive medium. With increasing incident light intensity above the saturation intensity, the shape becomes narrow. At the intensity of 0.7 $\times 10^{4} I_{s}$, the shape almost recovers its original shape which is expected without absorption. Figure 3(b) shows similar calculated curves of the coherent backscattering peaks for the sample of $l_{a}=100 l^{*}$. In this case, the shape of the coherent backscattering peak is not altered significantly as a function of the intensity of the incident light. The background intensity in Figs. 3(a) and 3(b) represents the reflectivity of the

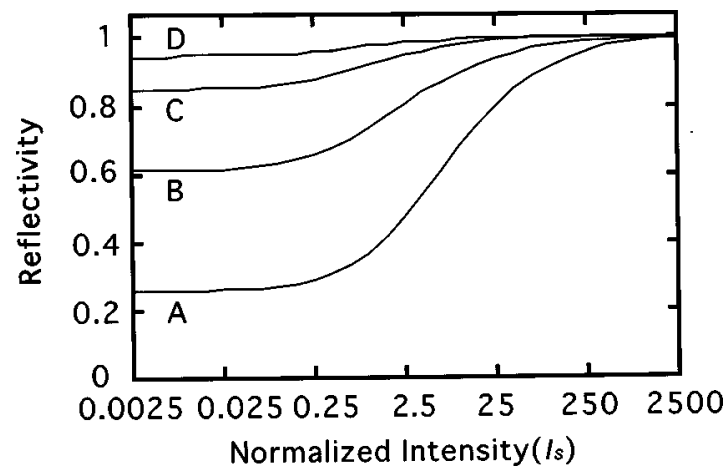

FIG. 4. Reflectivity of the incident light as a function of the excitation intensity. Solid lines are calculated for four different absorption lengths: (A) $l_{a}=2 l^{*}$; (B) $l_{a}=20 l^{*}$; (C) $l_{a}=200 l^{*}$; and (D) $l_{a}=2000 l^{*}$.

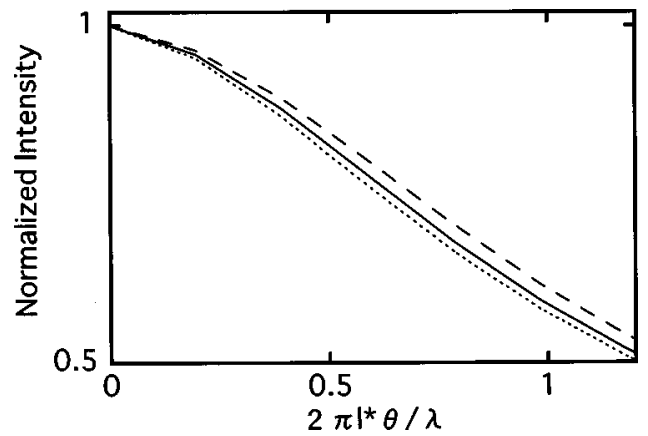

FIG. 5. Dotted and dashed lines are the coherent backscattering peaks for the normal absorption and the confined geometry, respectively. The solid line is the calculation for the saturation absorption. The dotted and solid lines are calculated on the condition $l_{a}$ $=8.6 l^{*}$ and $l_{a}=l^{*}$ with a semi-infinite size medium, respectively. In the calculation of the saturation absorption, the incident excitation light intensity is $0.7 \times 10^{3} I_{S}$. The dashed line is calculated on the sample with a thickness of $2.25 l^{*}$ with no absorption.

incident light. The increment in the backscattering intensity means that the reflectivity is increased with increasing the incident light intensity. The solid lines in Fig. 4 are the reflectivity of the incident light calculated using Eqs. (4) and (7) for different absorption lengths. From all the lines we understand that in low incident pulse energy, reflectivity decreases with increasing absorption. It starts to increase at the saturation intensity, and then converges to 1 at an incident intensity far larger than the saturation intensity.

We compare the effect of saturation absorption on the peak with the effects of the normal absorption and the confined geometry. Figure 5 shows the shape of the coherent backscattering peak for three cases. In all curves, the reflectively of the incident light is set to be 0.46 . This means that, for the finite slab, 54\% of the incident light will transmitted and, for the normal and saturation absorption, $54 \%$ of the incident light is absorbed, respectively. Etemad has discussed the difference of the effects of the finite slab and normal absorption on the shape of the peak. ${ }^{4}$ In the normal absorption all trajectories are terminated with a probability $\exp \left(-\eta / l_{a}\right)$, where $\eta$ is the length of the trajectories and $l_{a}$ is the absorption length. Therefore, the tails as well as the top of the peak is removed. On the other hand, in the case of the finite slab, the short trajectories with length is less than $w^{2} / l^{*}$, where $w$ is the thickness of the sample, are not subject to the effect of the confined geometry. Then the tails of the peak are not altered so much, while the top of the peak is removed. The effect of the saturation absorption to the shape of the peak is between two effects of the confined geometry and the normal absorption. At the surface region the absorption is saturated and absorption is very weak under the condition of the saturation absorption. The absorption appears as a step function at a certain distance from the surface which depends on the intensity. This suggests that the sample behaves like a slab. In the saturated region, the density of incident light in Fig. 1 decreases linearly, while in the normal absorption region the incident light decays as an exponential function. This results in that the effect of the saturation absorption is like the finite slab compared with the normal absorption.

It is interesting to consider numerical estimation for ex- 
perimental systems to observe the effect of the saturation absorption on the peak. A possible system may be a colloidal solution containing dye and $\mathrm{TiO}_{2}$ particles in methanol. When the $\mathrm{TiO}_{2}$ particles of $0.28 \mu \mathrm{m}$ in diameter are dispersed in methanol, the transport mean free path $l^{*}$ is $7 \mu \mathrm{m}$ with a particle density of $1 \times 10^{12} \mathrm{~cm}^{-3} \cdot{ }^{13}$ For rhodamine 640 dye with concentration $2 \times 10^{-2} \mathrm{M}$, the absorption length, $l_{a}$, is $7 \mu \mathrm{m}$, therefore $l^{*} \sim l_{a}$, which corresponds to the calculation shown in Fig. 3(a). The lifetime of rhodamine 640 is $2 \mathrm{~ns}$. With these parameters, the saturation intensity $I_{S}$ is estimated to be $4 \times 10^{28}\left(\mathrm{~m}^{-2} \mathrm{~s}^{-1}\right)$, which corresponds to $1 \times 10^{-5} \mathrm{~J}$ for the $532 \mathrm{~nm}$ pump pulse with $1 \mathrm{~ns}$ pulse width and $1 \mathrm{~mm}$ beam diameter. This excitation pulse energy could easily be obtained with a $Q$-switched $\mathrm{Nd}^{3+}$ YAG laser.

Zyuzin first discussed the shape of the coherent backscattering peak in the presence of amplification. ${ }^{14}$ Amplification could significantly alter the propagation of light in a disordered medium since the long paths are more amplified. Wiersma reported that the shape of the coherent backscattering peak becomes sharp in the optically pumped $\mathrm{TiAl}_{2} \mathrm{O}_{3}$ powder with amplification gain. ${ }^{10} \mathrm{~A}$ more interesting system may be dye-colloidal systems where the amplifying gain co- efficient is much higher. ${ }^{15}$ It has, however, been reported that in dye-colloidal system, the broad homogeneous broadening in the dye spectrum results in the saturation in the absorption not only at a wavelength of excitation light $(532 \mathrm{~nm})$ but also at the wavelength of probe beam $(620 \mathrm{~nm})$, when a strong excitation beam is incident on the system. ${ }^{13}$ It would be necessary to consider the saturation effect in the detailed analysis of the shape of the backscattering peak in this system.

In conclusion we have analyzed the reflected light from the disordered medium in the presence of saturation absorption. When the excitation intensity is increased above the saturation intensity, the reflectivity is increased and the width of the peak becomes narrow. This effect is evident when the absorption length becomes comparable to the transport mean free path. The effect of the saturation absorption could appear in a variety of phenomena in light propagation in the disordered medium, such as statistics of fluctuations, time spreading, and dynamic light scattering.

M.T. is grateful to Professor A. Lagendijk and Dr. G. Soest for stimulating discussions. The authors are also grateful to Dr. Matsumoto for his advice on the numerical calculations.
${ }^{1}$ M. P. van Albada and A. Lagendijk, Phys. Rev. Lett. 55, 2692 (1985).

${ }^{2}$ P.-E. Wolf and G. Maret, Phys. Rev. Lett. 55, 2696 (1985).

${ }^{3}$ E. Akkermans, P. E. Wolf, and R. Maynard, Phys. Rev. Lett. 56, 1471 (1986).

${ }^{4}$ S. Etemad, R. Thompson, and M. J. Andrejco, Phys. Rev. Lett. 57, 575 (1986).

${ }^{5}$ M. Kaveh, M. Rosenbluh, I. Edrei, and I. Freund, Phys. Rev. Lett. 57, 2049 (1986).

${ }^{6}$ I. Freund, M. Rosenbluh, R. Berkovits, and M. Kaveh, Phys. Rev. Lett. 61, 1214 (1988).

${ }^{7}$ R. Vreeker, M. P. van Albada, R. Sprik, and A. Lagendijk, Phys. Lett. A 132, 51 (1988).
${ }^{8}$ M. Tomita and H. Ikari, Phys. Rev. B 43, 3716 (1991).

${ }^{9}$ S. Etemad, R. Thompson, M. J. Andrejco, S. John, and F. C. MacKintosh, Phys. Rev. Lett. 59, 1420 (1987).

${ }^{10}$ D. S. Wiersma, M. P. van Albada, and A. Lagendijk, Phys. Rev. Lett. 75, 1739 (1995).

${ }^{11}$ A. Z. Genack and J. M. Drake, Nature (London) 368, 400 (1994).

${ }^{12}$ K. Totsuka, M. A. I. Talukder, and M. Tomita, J. Phys. Soc. Jpn. 68, 307 (1998).

${ }^{13}$ K. Totsuka, M. A. I. Talukder, M. Matsumoto, and M. Tomita, Phys. Rev. B 59, 50 (1999).

${ }^{14}$ A. Yu. Zyuzin, Europhys. Lett. 26, 517 (1994).

${ }^{15}$ P. C. de Oliveira, A. E. Perkins, and N. M. Lawandy, Opt. Lett. 21, 1685 (1996). 